Pacific Journal of Mathematics

SEPARABLE CONJUGATE SPACES 


\section{SEPARABLE CONJUGATE SPACES}

\section{ROBERT C. JAMES}

A Banach space $B$ is reflexive if the natural isometric mapping of $B$ into the second conjugate space $B^{* *}$ covers all of $B^{* *}$. All conjugate spaces of a reflexive separable space $B$ are separable. The nonreflexive space $l^{(1)}$ is separable and its first conjugate space is $(m)$, which is nonseparable. The space $\left(c_{0}\right)$ is separable, its first conjugate space is $l^{(1)}$, and its second conjugate space is $(m)$. An example is known of a nonreflexive Banach space whose conjugate spaces are all separable [4]. This space is pseudo-reflexive in the sense that its natural image in the second conjugate space has a finite-dimensional complement. The structure of such spaces has been studied carefully [2].

The main purpose of this paper is to show that the sequence started by $l^{(1)}$ and $\left(c_{0}\right)$ can be extended to give a sequence $\left\{B_{n}\right\}$ of separable Banach spaces such that, for each $n$, the $n$th conjugate space of $B_{n}$ is its first nonseparable conjugate space. The principal tool used is a theorem which states a sufficient condition on a space $T$ for the existence of a space $B$ with

$$
B^{* *}=\pi(B)+T,
$$

where $\pi(B)$ is the natural image of $B$ in $B^{* *}$. The following definition and notation will be used.

A basis for a Banach space $B$ is a sequence $\left\{u^{i}\right\}$ such that, for each $x$ of $B$, there is a unique sequence of numbers $\left\{a_{i}\right\}$ for which $\lim _{n \rightarrow \infty} \| x-$ $\sum_{1}^{n} a_{i} u_{i} \|=0$. A sequence $\left\{u_{i}\right\}$ is a basis for its closed linear span if and only if there is a number $\varepsilon>0$ such that

$$
\left\|\sum_{1}^{n+p} c_{i} x_{i}\right\| \geq \varepsilon\left\|\sum_{1}^{n} c_{i} x_{i}\right\|
$$

for any numbers $\left\{c_{i}\right\}$ and positive integers $n$ and $p[1$, page 111]. If $\varepsilon$ can be +1 , the basis is an orthogonal basis. It will be useful to classify bases as follows:

Type $\alpha$. If $\left\{a_{i}\right\}$ is a sequence of numbers for which $\sup _{n}\left\|\sum_{1}^{n} a_{i} u_{i}\right\|<$ $\infty$, then $\sum_{1}^{\infty} a_{i} u_{i}$ converges.

Type $\beta$. If $f$ is a linear functional defined on $B$ and $\|f\|_{n}$ is the norm of $f$ on the closed linear span of $\left\{u_{i} \mid i \geq n\right\}$, then $\lim _{n \rightarrow \infty}\|f\|_{n}=0$.

There are Banach spaces which have bases which are neither of type $\alpha$ nor of type $\beta$, while a basis is of both types if and only if the space

Reçẹved April 28, 1959. 
is reflexive [3; Theorem 1].

The symbols $C,(m), l^{(1)}$, and $\left(c_{0}\right)$ are used in the usual sense [1; pages 11, 12, 181]. The set of all $r+t$ with $r \in R$ and $t \in T$ is denoted by $R+T$. A space $R$ is said to be embedded in a space $S$ if $R$ is mapped isomorphically and isometrically on a subspace of $S$; for $x \in R$, the image of $x$ is indicated by $x^{(S)}$. In particular, $x^{(\sigma)}$ is a continuous function defined on $[0,1]$ and the value of $x^{(\theta)}$ at $t$ is denoted by $x^{(\theta)}(t)$. If $w=\left(w_{1}, w_{2}, \cdots\right)$ is a sequence of numbers, then ${ }^{n} w$ is the sequence obtained by replacing $w_{i}$ by 0 if $i>n$. A block of $w$ is a sequence ${ }_{m}^{n} w$ obtained from $w$ by replacing $w_{i}$ by 0 if $i \leq m$ or $i>n$. Two blocks ${ }_{m_{1}^{1}}^{n} w$ and ${ }_{m_{2}^{2}}^{n} w$ are said to overlap if the intervals $\left(m_{1}, n_{1}\right]$ and $\left(m_{2}, n_{2}\right]$ overlap.

Lemma 1. Let $T$ be a Banach space with an orthogonal basis $\left\{u_{i}\right\}$. Then $T$ can be embedded in $(m)$ in such a way that:

(i) if $x=\sum_{1}^{\infty} a_{i} u_{i}$, then the first $2 N$ coordinates of $x^{(m)}$ are zero if and only if $a_{i}=0$ for $i \leq N$;

(ii) if $\left\{a_{i}\right\}$ and $\left\{x_{i}^{m}\right\}$ are related by $x=\sum_{1}^{\infty} a_{i} u_{i}$ and $x^{(m)}=\left(x_{1}^{m}, x_{2}^{m}, \cdots\right)$, then $a_{1}, \cdots, a_{N}$ are each continuous functions of $x_{1}^{m}, \cdots, x_{2 N}^{m}$ and $x_{1}^{m}, \cdots, x_{2 N}^{m}$ are each continuous functions of $a_{1}, \cdots, a_{N}$;

(iii) if $x^{(m)}=\left(x_{1}^{m}, x_{2}^{m}, \cdots\right)$, then $\left\|x^{(m)}\right\|=\lim \sup \left|x_{i}^{m}\right|$.

Proof. Let $T$ be embedded in the space $C$. Let $\left\{t_{i}\right\}$ be a sequence of numbers in the interval $[0,1]$ for which the sequence $\left\{t_{2 i-1}\right\}, i=$ $1,2, \cdots$, is dense in $[0,1]$ and, for each $i, u_{i}^{(\sigma)}\left(t_{2 i}\right) \neq 0$. If $x=\sum_{1}^{\infty} a_{i} u_{i}$, let $x^{(m)}$ be the sequence $\left(x_{1}^{m}, x_{2}^{m}, \ldots\right)$ for which

$$
x_{2 k-1}^{m}=\sum_{i}^{k} a_{i} u_{i}^{(C)}\left(t_{2 k-1}\right), x_{2 k}^{m}=\sum_{1}^{k} a_{i} u_{i}^{(\sigma)}\left(t_{2 k}\right) .
$$

Then for any $t \in[0,1]$,

$$
\left|\sum_{1}^{k} a_{i} u_{i}^{(\sigma)}(t)\right| \leq\left\|\sum_{1}^{k} a_{i} u_{i}^{(\sigma)}\right\|=\left\|\sum_{1}^{k} a_{i} u_{i}\right\| \leq\|x\| .
$$

Hence $\left\|x^{(m)}\right\| \leq\|x\|$. But if $\varepsilon>0$ and $N$ is chosen so that $\left\|x-\sum_{1}^{k} \alpha u_{i}\right\|<\varepsilon$ if $k>N$, then it follows from $\left\{t_{2 k-1}\right\}$ being dense in $[0,1]$ that

$$
\left\|x^{(m)}\right\| \geq \sup _{k>N}\left|\sum_{1}^{k} a_{i} u_{i}^{(\sigma)}\left(t_{2 k-1}\right)\right| \geq\|x\|-\varepsilon .
$$

Hence $\|x\|=\left\|x^{(m)}\right\|$ and $T$ and its image in $(m)$ are isometric. But if $x=\sum_{N+1}^{\infty} a_{i} u_{i}$, then $x_{2 k-1}^{m}=x_{2 k}^{m}=0$ if $k \leq N$. If $x_{i}^{m}=0$ for $i \leq 2 N$, then the equations $x_{2 k}^{m}=\sum_{1}^{k} a_{i} u_{i}^{(O)}\left(t_{2 k}\right)=0, k \leq N$, successively imply $0=a_{1}=a_{2}=\cdots=a_{N}$, since $u_{k}^{(\sigma)}\left(t_{2 k}\right) \neq 0$. The conclusion (ii) follows from this system of equations and the continuity of $\sum_{1}^{N} a_{i} u_{i}$ in $a_{1}, \cdots, a_{N}$, while (iii) follows from $\left\{t_{2 \varepsilon-1}\right\}$ being dense in $[0,1]$. 
Lemma 2. Let $T$ be a Banach space with an orthogonal basis $\left\{u_{i}\right\}$ and let $T$ be embedded in $(m)$ as described in Lemma 1. Then the following are equivalent:

(i) the basis $\left\{u_{i}\right\}$ is of type $\alpha$;

(ii) if $w \in(m)$, then $w=v+t$, with $v$ an element of $(m)$ which has all coordinates zero after the Mth $(M \geq 0)$ and $t$ the image of an element of $T$, provided there is a sequence of elements $\left\{y_{k}\right\}$ of $T$ for which $\sup \left\|y_{k}\right\|<\infty$ and

$$
\lim _{k \rightarrow \infty} y_{k, i}^{m}=w_{i} \text { for } i>M,
$$

where $w=\left(w_{1}, w_{2}, \cdots\right)$ and $y_{k}^{(m)}=\left(y_{k, 1}^{m}, y_{k, 2}^{m}, \cdots\right)$.

Proof. Assume the basis $\left\{u_{i}\right\}$ is of type $\alpha$ and let $w=\left(w_{i}, w_{2}, \cdots\right)$ and $\left\{y_{k}\right\}$ satisfy the hypotheses of (ii). Since $\left\|y_{k}\right\|$ is bounded, there is a subsequence $\left\{z_{k}\right\}$ of $\left\{y_{k}\right\}$ such that

$$
\lim _{k \rightarrow \infty} z_{k, i}^{m}=v_{i}
$$

exists for $i \leq M$. Let $v=\left(w_{1}-v_{1}, \cdots, w_{M}-v_{M}, 0,0, \cdots\right)$. Also let $z_{k}=\sum_{1}^{\infty} a_{i}^{k} u_{i}$ for each $k$. It now follows from (ii) of Lemma 1 that $\lim _{k \rightarrow \infty} a_{i}^{k}=a_{i}$ exists for each $i$. Since the basis is orthogonal, $\left\|\sum_{1}^{n} a_{i} u_{i}\right\| \leq$ $\sup \left\|z_{k}\right\|$. Since $\left\{u_{i}\right\}$ is a basis of type $\alpha$, it then follows that $\sum_{1}^{\infty} a_{i} u_{i}$ is convergent. Also, $w-v=t$ is the $(m)$-image of $\sum_{1}^{\infty} a_{i} u_{i}$. This follows from the fact that the numbers $a_{i}, i \leq N$, continuously determine the first $2 N$ coordinates of the $(m)$-image of $\sum_{1}^{\infty} a_{i} u_{i}$, while $z_{k}=\sum_{1}^{\infty} a_{i}^{k} u_{i}$, $\lim _{k \rightarrow \infty} a_{i}^{k}=\alpha_{i}$, and $\lim _{k \rightarrow \infty} z_{k, i}^{m}$ exists and is the $i$ th coordinate of $w-v$.

Now assume (ii) and let $\left\|\sum_{1}^{n} a_{i} u_{i}\right\|$ be a bounded function of $n$. Let $w=\left(w_{1}, w_{2}, \cdots\right)$ be the element of $(m)$ whose first $2 N$ coordinates are determined by $a_{1}, \cdots, a_{N}$. Take $M=0$ and $y_{k}$ to be the $(m)$-image of $\sum_{1}^{k} a_{i} u_{i}$. It then follows from (ii) that $w$ is the $(m)$-image of some element of $T$, which can only be $\sum_{1}^{\infty} a_{i} u_{i}$.

THEOREM 1. Let $T$ be a Banach space which has an orthogonal basis of type $\alpha$. Then there is a Banach space $B$ which has a basis of type $\beta$ and for which

$$
B^{* *}=\pi(B)+T_{1},
$$

where $\pi(B)$ is the natural image of $B$ in $B^{* *}$, $T$ and $T_{1}$ are isometric, and $\|r+t\| \geq\|t\|$ if $r \in \pi(B)$ and $t \in T_{1}$.

Proof. Let $T_{1}$ be the embedding of $T$ in $(m)$ as described in Lemma 1. The norm of $(m)$ will be denoted by \|\| . For elements $w$ of $(m)$ which have only a finite number of nonzero coordinates, let

(1) $\theta(w)=\inf \|t\|$ for $w$ a block of $t$, where $t$ is either a member 
of $T_{1}$ or has only one nonzero coordinate (note that $\theta(w)$ is defined only for elements $w$ which are blocks of at least one $t \in T_{1}$ or which have only one nonzero coordinate);

(2) $h(w)=\left\{\inf \sum\left[\theta\left(b_{i}\right)\right]^{2}\right\}^{1 / 2}$, where $w=\sum b_{i}$, each $b_{i}$ is a block of $w$, and no two blocks overlap.

(3) $\quad\|x\|=\inf \sum h\left(w_{j}\right)$ for $x=\sum w_{j}$.

In the above, all sums have a finite number of terms. The triangular inequality for $\||\||$ is a direct consequence of (3). Also, $\| x \| \geq \geq$ $\|x\|$, since $\theta(w) \geq\|w\|$ and $h(w) \geq\|w\|$. Let $B$ be the completion of the space of sequences with a finite number of nonzero coordinates, using the norm \|\|$\|$. The sequence of elements $\left\{u_{i}\right\}$ for which $u_{i}$ has all coordinates 0 except the $i$ th, which is 1 , is an orthogonal basis for $B$. This means that $\left\|\left|\sum_{1}^{n+p} a_{i} u_{i}\right||\geq||| \sum_{1}^{n} a_{i} u_{i}|\||\right.$, which follows by noting that, if $\sum_{1}^{n+p} a_{i} u_{i}=\sum w_{j}$, then $\sum_{1}^{n} a_{i} u_{i}=\sum^{n} w_{j}$ and $h\left({ }^{n} w_{j}\right) \leq h\left(w_{j}\right)$ for each $j$, where ${ }^{n} w_{j}$ is obtained from $w_{j}$ by replacing each coordinate after the $n$th by 0 .

The basis $\left\{u_{i}\right\}$ is of type $\beta$. For suppose there is a linear functional $f$ for which $\lim _{n \rightarrow \infty}\left|\|f \mid\|_{n}=K \neq 0\right.$ and choose $N$ so that $\|f \mid\|_{N} \leq 7 / 6 K$. Then there are two elements $x=\sum_{n_{1}^{2}}^{n_{2}} a_{i} u_{i}, y=\sum_{n_{3}^{4}}^{n_{4}} a_{i} u_{i}$, for which $N<$ $n_{1} \leq n_{2}<n_{3} \leq n_{4},\|x\|=\|\| y \|=1, f(x)>7 / 8 K$ and $f(y)>7 / 8 K$. Then

$$
\frac{7}{4} K<f(x)+f(y) \leq\left(\frac{7}{6} K\right)\|\| x+y \| \mid \text { and }\|x+y\|>\frac{3}{2} .
$$

Since $\theta$ and $h$ are both monotone decreasing as a block has coordinates at the ends replaced by zeros, there exists $\left\{x_{j}\right\}$ and $\left\{y_{j}\right\}$ such that $x=\sum x_{j}, y=\sum y_{j}, \sum h\left(x_{j}\right)<\|x\|+\varepsilon$, and $\sum h\left(y_{j}\right)<\|y\|+\varepsilon$, where each $x_{j}$ has zero coordinates outside the index interval $\left[n_{1}, n_{2}\right]$ and each

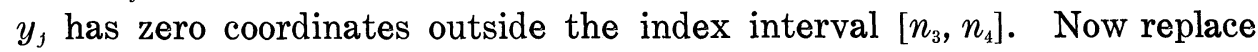
the sets $\left\{x_{j}\right\}$ and $\left\{y_{j}\right\}$ by $\left\{\bar{x}_{j}\right\}$ and $\left\{\bar{y}_{j}\right\}$ defined as follows: if $h\left(x_{p}\right)$ is the smallest of all the numbers $h\left(x_{j}\right)$ and $h\left(y_{j}\right)$, then let $\bar{x}_{1}=x_{p}$ and $\bar{y}_{1}=\left[h\left(x_{p}\right) / h\left(y_{r}\right)\right] y_{r}$ (for some $r$ ) and replace $y_{r}$ by $\left[1-h\left(x_{p}\right) / h\left(y_{r}\right)\right] y_{r}$. The analogous process is used if $h$ takes on its minimum at one of the $y_{j}$ 's. This process creates two new elements and eliminates one old one at each step, until all of the $x_{j}$ 's or all of the $y_{j}$ 's are eliminated. If only $x_{j}$ 's remain, say $x_{p_{j}}$ 's, then $\sum h\left(x_{p_{j}}\right)<\varepsilon$, and similarly $\sum h\left(y_{p_{j}}\right)<\varepsilon$ if only $y_{j}$ 's remain. Also

$$
\sum h\left(\bar{x}_{j}\right)-\varepsilon=\sum h\left(\bar{y}_{j}\right)-\varepsilon<\|\| x\|=\| y \|=1
$$

and $h\left(\bar{x}_{j}\right)=h\left(\bar{y}_{j}\right)$ for each $j$. For each $j$, there are nonoverlapping blocks $\left\{\bar{x}_{j i}\right\}$ and $\left\{\bar{y}_{j i}\right\}$ such that

$$
h\left(\bar{x}_{j}\right)=h\left(\bar{y}_{j}\right)=\left\{\sum_{i}\left[\theta\left(\bar{x}_{1 i}\right)\right]^{2}\right\}^{1 / 2}=\left\{\sum_{i}\left[\theta\left(\bar{y}_{j i}\right)\right]^{2}\right\}^{1 / 2} .
$$

Then 


$$
\left.h\left(\bar{x}_{j}+\bar{y}_{j}\right) \leq\left\{\sum_{i}\left[\theta \bar{x}_{j i}\right)\right]^{2}+\sum_{i}\left[\theta\left(\bar{y}_{j i}\right)\right]^{2}\right\}^{1 / 2}=\sqrt{\overline{2}} h\left(\bar{x}_{j}\right) .
$$

Hence

$$
\|x+y\| \leq \sum h\left(\bar{x}_{j}+\bar{y}_{j}\right)+\varepsilon \leq \sqrt{2} \sum h\left(\bar{x}_{j}\right)+\varepsilon \leq \sqrt{2}+\varepsilon .
$$

Since $\|x+y\| \mid>3 / 2$, this is contradictory if $\sqrt{2}+\varepsilon<3 / 2$. It has therefore been shown that $\left\{u_{i}\right\}$ is a basis of type $\beta$.

Since $\left\{u_{i}\right\}$ is an orthogonal basis of type $\beta$ for $B$, it follows that $B^{* *}$ consists of all sequences $F=\left(F_{1}, F_{2}, \cdots\right)$ for which

$$
\|F\|=\lim _{n \rightarrow \infty}\left|\left\|\left(F_{1}, \cdots, F_{n}, 0,0, \cdots\right)\right\|\right|
$$

exists [4; page 174]. Note first that if $t=\left(t_{1}, \cdots\right) \in T_{1}$, then

$$
\left\|\mid\left(t_{1}, \cdots, t_{n}, 0,0, \cdots\right)\right\|\|=\|\left(t_{1}, \cdots, t_{n}, 0,0, \cdots\right) \|
$$

and $\lim _{n \rightarrow \infty} \mid\left\|\left(t_{1}, \cdots, t_{n}, 0,0, \cdots\right)\right\|=\|t\|=\|t\|$. Thus $T_{1} \subset B^{* *}$. Also, the natural mapping of $B$ into $B^{* *}$ is merely the mapping of a sequence in $B$ onto the identical sequence in $B^{* *}$. It then follows that $\|r+t\| \| \geq$ $\|t\|$ if $r \in \pi(B)$ and $t \in T_{1}$, since $r$ can be approximated by a sequence with a finite number of nonzero coordinates but (Lemma 1) $\|t\|=$ $\lim \sup \left|t_{i}\right|$.

Now suppose that $F=\left(F_{1}, F_{2}, \cdots\right)$ is a sequence for which $\lim _{n \rightarrow \infty}\left|\left\|{ }^{n} F \mid\right\|\right.$ exists; i.e., $F \in B^{* *}$. It will be shown that there is an element $v$ of $\pi(B)+T_{1}$ for which $\||F-v\||| \leq 15 / 16\|||F|\|$. Successive application of this would then establish that $F \in \pi(B)+T_{1}$. For each $n$, there are ${ }^{n} w_{j}$ and blocks $b_{j, i}^{n}$, which are either blocks of elements of $T_{1}$ or have only one nonzero coordinate, such that

$$
\left|\left\|{ }^{n} F \mid\right\|=\sum_{j} h\left({ }^{n} w_{j}\right),{ }^{n} F=\sum_{j}{ }^{n} w_{j} \text {, and } h\left({ }^{n} w_{j}\right)=\left\{\sum_{i}\left[\theta\left(b_{j, i}^{n}\right)\right]^{2}\right\}^{1 / 2},\right.
$$

where each ${ }^{n} w_{j}$ and each $b_{j, i}^{n}$ have all coordinates zero after the $n$ th. This follows by a limit argument, using the facts (1) that there are only a finite number $K_{n}$ of ways of choosing division points for nonoverlapping blocks from the integers $1,2, \cdots, n$ and (2) that it follows from Lemma 1 and the orthogonality of the basis for $T$ that $\theta\left(b_{j, i}^{2 N}\right)$, for a block $b_{j, i}^{2 N}$ which has zero coordinates beyond the $2 N$ th coordinate, can be evaluated by using only members of the span of the first $N$ basis elements of $T$.

If $m<n$ and ${ }^{m} w_{j}^{n}$ is obtained from ${ }^{n} w_{j}$ by replacing coordinates after the $m$ th by zeros, then

$$
\left\|{ }^{m} F\right\| \leq \sum_{j} h\left({ }^{m} w_{j}^{n}\right) \leq\left\|{ }^{n} F \mid\right\| \leq\|F\| .
$$

If ${ }^{m} w_{j_{1}}^{n}$ and ${ }^{m} w_{j_{2}}^{n}$ are of the "same type" in the sense that they are divided into blocks by using the same division points, then it follows by using these same division points for ${ }^{m} w_{j_{1}}^{n}+{ }^{m} w_{j_{2}}^{n}$ that 


$$
h\left({ }^{m} w_{j_{1}}^{n}+{ }^{m} w_{j_{2}}^{n}\right) \leq h\left({ }^{m} w_{j_{1}}^{n}\right)+h\left({ }^{m} w_{j_{2}}^{n}\right) .
$$

For each $n>m$, let ${ }^{m} \hat{w}_{j}^{n}$ be the sum of all ${ }^{m} w_{j_{i}}^{n}$ of the "same type" as ${ }^{m} \hat{w}_{j}^{n}$. A limit argument gives a sequence of integers $\left\{n_{i}\right\}$ such that $\lim { }^{m} \hat{w}_{J}^{n_{i}}={ }^{m} \bar{w}_{j}$ exists for each "type". If $m<n$, then there exist $\bar{b}_{j, i}^{n}$ such that

$$
\begin{aligned}
\left\|{ }^{m} \boldsymbol{F}\right\| & \leq \sum_{j} h\left(^{m} \bar{w}_{j}\right) \leq \sum_{k} h\left({ }^{n} \bar{w}_{k}\right) \leq\|\| \boldsymbol{F}\|\|, \\
h\left({ }^{m} \bar{w}_{j}\right) & =\left\{\sum_{i}\left[\theta\left(\bar{b}_{j, i}^{m}\right)\right]^{2}\right\}^{1 / 2},{ }^{m} \boldsymbol{F}=\sum^{m} \bar{w}_{j},
\end{aligned}
$$

and ${ }^{m} \bar{w}_{j}$ is equal to the sum of all ${ }^{m} \bar{w}_{j}^{n}$ which are of the same type as ${ }^{n} \bar{w}_{j}$ and are obtained from ${ }^{n} \bar{w}_{j}$ by replacing all coordinates after the $m$ th by zeros. The points used to divide ${ }^{m} \bar{w}_{j}$ into the blocks $\bar{b}_{j, i}^{m}$ will be called the division points of ${ }^{m} \bar{w}_{j}$.

Choose $M$ so that ||${ }^{m} F\left|\|>15 / 16|||F|\|\right.$. Note that if ${ }^{m} \bar{w}_{j}$ is of a particular type and $n>m$, then ${ }^{m} \bar{w}_{\text {, }}$ is the sum of one or more elements obtained from the ${ }^{n} \bar{w}_{k}$ 's by replacing coordinates after the $m$ th by zeros. For $n>m \geq M$, let ${ }^{n} t$ be the sum of all ${ }^{n} \bar{w}_{k}$ 's which have no division points between $M$ and $n$ and let ${ }^{m} t^{n}$ be obtained from ${ }^{n} t$ by replacing coordinates after the $m$ th by zeros. Let $\left\{n_{i}\right\}$ be chosen so that

$$
\lim _{i \rightarrow \infty}{ }^{m} t^{n i}={ }^{m} \bar{t}
$$

exists for each $m \geq M$. Let $\bar{t}$ be defined so as to have the same first $m$ coordinates as ${ }^{m} \bar{t}$. Then any finite block of $\bar{t}$ whose first $M$ coordinates are zero is also approximately a block of an element of $T_{1}$ and these elements of $T_{1}$ are of bounded norm. It then follows from Lemma 2 that there is an element $v_{0}$, with a finite number of nonzero coordinates, such that $v_{0}+\bar{t} \in T_{1}$. Thus

$$
\bar{t} \in \pi(B)+T_{1} .
$$

First assume that $\||\bar{t}|||>1 / 8|\| F|||$ and choose $N$ so that

$$
\|{ }^{n} \bar{t}||>1 / 8|| F|| \text { if } n>N \text {. }
$$

For $n>N$, choose $p>n$ so that

$$
\left|\| \bar{n} \bar{t}-{ }^{n} t^{p}\right|\left|<\frac{1}{32}\right||F||| .
$$

Since ||${ }^{n} F \mid \| \leq \sum_{j} h\left({ }^{n} \bar{w}_{j}\right)$, discarding all ${ }^{n} \bar{w}_{j}^{p}$ without division points between $M$ and $p$ gives

$$
\begin{aligned}
\|{ }^{n} F-{ }^{n} t^{p}|| \mid & \leq \sum h\left({ }^{n} \bar{w}_{j}\right)-\left\|{ }^{n} t^{p}|\||\right. \\
& \leq \||| F||-||{ }^{n} t^{p}|| \mid .
\end{aligned}
$$


Hence ||${ }^{n} F-{ }^{n} \bar{t}||<|||F|||-||{ }^{\bar{t}}|||+1 / 16\||F||<15 / 16 \|| F||$. Since $n$ was an arbitrary integer with $n>N$, it follows that

$$
\||F-\bar{t}|\| \leq \frac{15}{16}|| F \mid \| \text {. }
$$

Now assume that $\|\bar{t}|\|\leq 1 / 8\|| F \mid\|$. Then $\left\|{ }^{n} \bar{t}\right\||\leq 1 / 8 \|||F| \mid$ for all $n$. Choose $q$ so that

$$
\left\|{ }^{M} \bar{t}-{ }^{M} t^{q}\left|\left\|<\frac{1}{16}\right\| F\right|\right\| .
$$

For each ${ }^{q} \bar{w}_{j}$ which has a division point between $M$ and $q$, let $u_{j}^{q}$ be obtained from ${ }^{a} \bar{w}_{j}$ by replacing all coordinates after the last such division point by zeros. Let

$$
u=\sum_{j} u_{j}^{q} .
$$

Choose $n>q$. Then ${ }^{n} F=\sum^{n} \bar{w}_{j}$ and

$$
\begin{aligned}
\left\|{ }^{M} F\right\| \| & \leq \sum h\left({ }^{M} \bar{w}_{j}^{n}\right) \leq \sum h\left(u_{j}^{q}\right)+\left\|{ }^{M} t^{q}\right\| \| \\
& <\sum h\left(u_{j}^{q}\right)+\frac{3}{16}\|F\| .
\end{aligned}
$$

Since $\left|\left\|{ }^{M} F\right\|\right|\left|>15 / 16\||| F \mid\|\right.$, we have $\sum h\left(u_{j}^{q}\right)>3 / 4\||| F \mid\|$. Now consider $F-u$. Since $\left\|{ }^{n} F\right\| \leq \sum h\left({ }^{n} \bar{w}_{j}\right)$, where $h\left({ }^{n} \bar{w}_{j}\right)=\left\{\sum_{i}\left[\theta\left(b_{j, i}^{n}\right)\right]^{2}\right\}^{1 / 2}$, we have

$$
\begin{gathered}
{ }^{n}(F-u)=\sum^{n} \bar{w}_{j}-\sum u_{j}^{q}=\sum^{n} \tilde{w}_{j}, \\
\left\|{ }^{n}(F-u)\right\| \leq \sum h\left({ }^{n} \tilde{w}_{j}\right),
\end{gathered}
$$

where ${ }^{n} \widetilde{w}_{j}$ is obtained from ${ }^{n} \bar{w}_{j}$ by replacing all coordinates before the last division point between $M$ and $q$ by zeros (if there is no such point, then $\left.{ }^{n} \tilde{w}_{j}={ }^{n} \bar{w}_{j}\right)$. The following trivial facts will be used: If $A$ and $B$ are nonnegative and

$$
\begin{aligned}
& \text { if } \sqrt{3} A<B \text {, then } \sqrt{A^{2}+B^{2}}>2 A \\
& \text { if } \sqrt{3} A \geq B \text {, then } B<\sqrt{A^{2}+B^{2}}-\frac{1}{4} A .
\end{aligned}
$$

Each ${ }^{n} \bar{w}_{3}$ which has a division point between $M$ and $q$ makes a contribution to some $u_{j}^{q}$. For such an ${ }^{n} \bar{w}_{j}$, let

$$
h\left({ }^{n} \bar{w}_{j}\right)=\left[\sum_{r}\left(A_{r}\right)^{2}+\sum_{s}\left(B_{s}\right)^{2}\right]^{1 / 2},
$$

where the $A_{r}$ 's and $B_{r}$ 's are, respectively, the values of $\theta\left(\bar{b}_{j, i}^{n}\right)$ for $\bar{b}_{j, i}^{n}$ a block of some $u_{j}^{q}$ and $\bar{b}_{j, i}^{n}$ not a block of any $u_{j}^{q}$. Then 


$$
h\left(u_{j}^{q}\right) \leq \sum\left[\sum_{r}\left(A_{r}\right)^{2}\right]^{1 / 2},
$$

where the sum is over all ${ }^{n} \bar{w}_{j}$ which make a contribution to $u_{j}^{q}$. Let $\sum_{r}\left(A_{r}\right)^{2}$ be of class (1) or of class (2) according as

$$
\sqrt{3}\left[\sum\left(A_{r}\right)^{2}\right]^{1 / 2}<\left[\sum\left(B_{s}\right)^{2}\right]^{1 / 2} \text { or } \sqrt{3}\left[\sum\left(A_{r}\right)^{2}\right]^{1 / 2} \geq\left[\sum\left(B_{s}\right)^{2}\right]^{1 / 2} \text {. }
$$

Since $\sum h\left(u_{f}^{q}\right)>3 / 4|| F \mid \|$, the sum of all terms of class (1) is not larger than $1 / 2|||F|||$ (otherwise we would have $\sum h\left({ }^{n} \bar{w}_{j}\right)>|\|F \mid\|$ ) and the sum of all terms of class (2) is greater than $1 / 4|| F \mid \|$. But for a term of class (2),

$$
\left[\sum\left(B_{s}\right)^{2}\right]^{1 / 2}<h\left({ }^{n} \bar{w}_{j}\right)-\frac{1}{4}\left[\sum\left(A_{r}\right)^{2}\right]^{1 / 2}
$$

Adding these inequalities for each ${ }^{n} \bar{w}_{j}$ and discarding each $\sum\left(A_{r}\right)^{2}$ which is of class (1) gives

$$
\sum h\left({ }^{n} \tilde{w}_{j}\right)<\sum h\left({ }^{n} \bar{w}_{j}\right)-\frac{1}{16}\|F\| \mid \text { and }\left\|{ }^{n}(F-u)\right\| \mid<\frac{15}{16}\|F\| .
$$

Since $n$ was an arbitrary integer with $n>q$, it follows that

$$
\|F-u\|\left|\leq \frac{15}{16}\|F\|\right|
$$

The importance of the assumption in Theorem 1 that $T_{1}$ have a basis of type $\alpha$ is made clear by the fact that the theorem breaks down if $T_{1}$ has a subspace isomorphic with $\left(c_{0}\right)$. In fact, in this case there can not be a separable space $B$ with

$$
B^{* *}=\pi(B)+T_{1}
$$

and $T_{1}$ separable, whether or not $B$ and $T_{1}$ have bases. This follows from the fact that if a conjugate space $R^{*}$ contains a subspace isomorphic with $\left(c_{0}\right)$, then $R^{*}$ contains a subspace isomorphic with $(m)$ and is not separable. To establish this fact, suppose that $\left\{F_{n}\right\}$ are continuous linear functionals defined on some Banach space $B$ and that the closed linear span of $\left\{F_{n}\right\}$ is isomorphic with $\left(c_{0}\right)$, the correspondence being

$$
\sum_{1}^{\infty} a_{i} F_{i} \leftrightarrow\left(a_{1}, a_{2}, \cdots\right) .
$$

For any bounded sequence $w=\left(w_{1}, w_{2}, \cdots\right)$, define $F_{w}$ by

$$
F_{w}(f)=\lim _{n \rightarrow \infty}\left(\sum_{1}^{n} w_{i} F_{i}\right)(f),
$$

for each $f$ of $B$. This limit exists, since if it did not there would exist 
$\varepsilon>0$ and $G_{1}=\sum_{1}^{n_{1}} w_{i} F_{i}, G_{2}=\sum_{n_{2}^{3}}^{n_{3}} w_{i} F_{i}, \cdots$, with $1 \leq n_{1}<n_{2} \leq n_{3}<n_{4} \leq \cdots$, such that $G_{i}(f)>\varepsilon$. Then correct choice of signs would give

$$
\sum_{1}^{n} \pm G_{i}(f)>n \varepsilon,
$$

which contradicts the boundedness of $\left\|\sum^{n} \pm G_{i}\right\|$. Clearly the correspondence with $\left(c_{0}\right)$ is thus extended to a bicontinuous correspondence with $(m)$.

THEOREM 2. For any positive integer n, there is a Banach space $B_{n}$ such that the nth conjugate space of $B_{n}$ is the first nonseparable conjugate space of $B_{n}$.

Proof. Let $B_{1}=l^{(1)}$ and $B_{2}=\left(c_{0}\right)$. Then $B_{1}$ has a basis of type $\alpha$ and $B_{2}$ has a basis of type $\beta$. In the following, the notation $R+S$ is used only if $\|r+s\| \geq\|s\|$ whenever $r \in R$ and $s \in S$. It follows from Theorem 1 that there is a separable Banach space $B_{3}$ with a basis of type $\beta$ for which

$$
B_{3}^{* *}=B_{3}+l^{(1)}=B_{3}+B_{2}^{*}
$$

Then $B_{3}^{* * *}$ is nonseparable and $B_{3}^{*}$ has a basis of type $\alpha$ [3, Theorem 3]. Now suppose that, for $k \leq n, B_{k}$ has been found for which

$$
B_{k}^{k *}=B_{k}+B_{k-1}^{*}
$$

if $k \geq 3, B_{k}$ has a basis of type $\beta$ if $k \geq 2$, and the $k$ th conjugate space of $B_{k}$ is the first nonseparable conjugate space of $B_{k}$. Then $B_{n}^{*}$ has a basis of type $\alpha$ and it follows from Theorem 1 that there exists a separable space $B_{n+1}$ which has a basis of type $\beta$ and for which

$$
B_{n+1}^{* *}=B_{n+1}+B_{n}^{*} \text {. }
$$

Then $B_{n+1}^{* * *}=B_{n+1}^{*}+B_{n}+B_{n-1}^{*}$. The $(n-2)$ nd conjugate space of $B_{n-1}^{*}$ is the first nonseparable conjugate space of $B_{n-1}^{*}$, while the $(n-2)$ nd conjugate space of $B_{n}$ is separable. Hence the $(n+1)$ st conjugate space of $B_{n+1}$ is the first nonseparable conjugate space of $B_{n+1}$.

\section{REFERENCES}

1. S. Banach, Théorie des opérations linéaires, Warsaw, 1932.

2. P. Civin and B. Yood, Quasi-reflexive spaces, Proc. Amer. Math. Soc., 8 (1957), 906911.

3. R. C. James, Bases and reflexivity of Banach spaces, Annals of Math., 52 (1950), $518-527$.

4. - A non-reflexive Banach space isometric with its second conjugate space, Proc. Nat. Acad. Sci. U.S.A., 37 (1951), 174-177. 



\section{PACIFIC JOURNAL OF MATHEMATICS}

\section{EDITORS}

David Gilbarg

Stanford University

Stanford, California

F. H. Brownell

University of Washington

Seattle 5, Washington
A. L. Whiteman

University of Southern Californıa Los Angeles 7, California

L. J. PaIge

University of California

Los Angeles 24, California

\section{ASSOCIATE EDITORS}
E. F. BECKENBACH
T. M. CHERRY
D. DERRY

E. HEWITT
A. HORN
L. NACHBIN
M. OHTSUKA

H. L. ROYDEN

M. M. SCHIFFER
E. SPANIER

E. G. STRAUS

F. WOLF

\section{SUPPORTING INSTITUTIONS}

\author{
UNIVERSITY OF BRITISH COLUMBIA \\ CALIFORNIA INSTITUTE OF TECHNOLOGY \\ UNIVERSITY OF CALIFORNIA \\ MONTANA STATE UNIVERSITY \\ UNIVERSITY OF NEVADA \\ NEW MEXICO STATE UNIVERSITY \\ OREGON STATE COLLEGE \\ UNIVERSITY OF OREGON \\ OSAKA UNIVERSITY \\ UNIVERSITY OF SOUTHERN CALIFORNIA
}

\author{
STANFORD UNIVERSITY \\ UNIVERSITY OF TOKYO \\ UNIVERSITY OF UTAH \\ WASHINGTON STATE COLLEGE \\ UNIVERSITY OF WASHINGTON \\ AMERICAN MATHEMATICAL SOCIETY \\ CALIFORNIA RESEARCH CORPORATION \\ HUGHES AIRCRAFT COMPANY \\ SPACE TECHNOLOGY LABORATORIES \\ NAVAL ORDNANCE TEST STATION
}

Mathematical papers intended for publication in the Pacific Journal of Mathematics should be typewritten (double spaced), and the author should keep a complete copy. Manuscripts may be sent to any one of the four editors. All other communications to the editors should be addressed to the managing editor, L. J. Paige at the University of California, Los Angeles 24, California.

50 reprints per author of each article are furnished free of charge; additional copies may be obtained at cost in multiples of 50 .

The Pacific Journal of Mathematics is published quarterly, in March, June, September, and December. The price per volume (4 numbers) is $\$ 12.00$; single issues, $\$ 3.50$. Back numbers are available. Special price to individual faculty members of supporting institutions and to individual members of the American Mathematical Society: $\$ 4.00$ per volume; single issues, $\$ 1.25$.

Subscriptions, orders for back numbers, and changes of address should be sent to Pacific Journal of Mathematics, 2120 Oxford Street, Berkeley 4, California.

Printed at Kokusai Bunken Insatsusha (International Academic Printing Co., Ltd.), No. 6, 2-chome, Fujimi-cho, Chiyoda-ku, Tokyo, Japan.

PUBLISHED BY PACIFIC JOURNAL OF MATHEMATICS, A NON-PROFIT CORPORATION

The Supporting Institutions listed above contribute to the cost of publication of this Journal, but they are not owners or publishers and have no responsibility for its content or policies. 


\section{Pacific Journal of Mathematics}

\section{Vol. 10, No. $2 \quad$ October, 1960}

Maynard G. Arsove, The Paley-Wiener theorem in metric linear spaces ........

Robert (Yisrael) John Aumann, Acceptable points in games of perfect

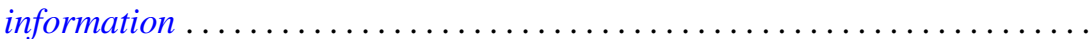

A. V. Balakrishnan, Fractional powers of closed operators and the semigroups generated by them ... . . . . . . . . . . . . . . . . . . . . . . . . . . . . 419

Dallas O. Banks, Bounds for the eigenvalues of some vibrating systems . . . . . 439

Billy Joe Boyer, On the summability of derived Fourier series . . . . . . . . . . . 475

Robert Breusch, An elementary proof of the prime number theorem with

remainder term ...................................

Edward David Callender, Jr., Hölder continuity of $n$-dimensional

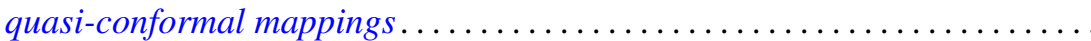

L. Carlitz, Note on Alder's polynomials ......................... 517

P. H. Doyle, III, Unions of cell pairs in $E^{3} \ldots \ldots \ldots \ldots \ldots \ldots \ldots \ldots \ldots \ldots \ldots \ldots . \ldots 21$

James Eells, Jr., A class of smooth bundles over a manifold . . . . . . . . . . . . 525

Shaul Foguel, Computations of the multiplicity function . . . . . . . . . . . . . . 539

James G. Glimm and Richard Vincent Kadison, Unitary operators in

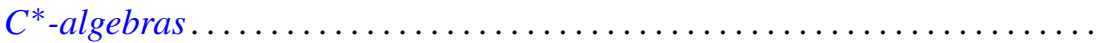

Hugh Gordon, Measure defined by abstract $L_{p}$ spaces . . . . . . . . . . . 557

Robert Clarke James, Separable conjugate spaces ....................

William Elliott Jenner, On non-associative algebras associated with bilinear forms

Harold H. Johnson, Terminating prolongation procedures

John W. Milnor and Edwin Spanier, Two remarks on fiber homotopy type .

Donald Alan Norton, A note on associativity . .

Ronald John Nunke, On the extensions of a torsion module.

Joseph J. Rotman, Mixed modules over valuations rings . . . . .

A. Sade, Théorie des systèmes demosiens de groupoï des . .

Wolfgang M. Schmidt, On normal numbers . .

661

Berthold Schweizer, Abe Sklar and Edward Oakley Thorp, The metrization of

statistical metric spaces

John P. Shanahan, On uniqueness questions for hyperbolic differential

equations

A. H. Stone, Sequences of coverings

Edward Oakley Thorp, Projections onto the subspace of compact operators

L. Bruce Treybig, Concerning certain locally peripherally separable spaces

Milo Wesley Weaver, On the commutativity of a correspondence and a

permutation

David Van Vranken Wend, On the zeros of solutions of some linear complex

differential equations. 